\title{
Acute aortic dissection: The conundrum of severity of disease, extent of surgery and end-organ function
}

\author{
Luca Salvatore De Santo, MD, FESC
}

\author{
From the University of Foggia, Foggia, Italy and Cardiac Surgery Unit, Casa di Cura Montevergine, GVM Care \\ and Research, Mercogliano, Italy. \\ Disclosures: Author has nothing to disclose with regard to commercial support. \\ Received for publication Jan 25, 2018; accepted for publication Jan 31, 2018; available ahead of print March 9 , \\ 2018. \\ Address for reprints: Luca Salvatore De Santo, MD, FESC, viale Colli Aminei 491, 80131 Naples, Italy (E-mail: \\ luca.desanto@unifg.it). \\ J Thorac Cardiovasc Surg 2018;155:2275-6 \\ $0022-5223 / \$ 36.00$ \\ Copyright (c) 2018 by The American Association for Thoracic Surgery \\ https://doi.org/10.1016/j.jtcvs.2018.01.063
}

Despite considerable improvements, management of type A acute aortic dissection (TA-AAD) remains challenging, and the optimal surgical strategy remains a topic of debate. Excision of the primary entry tear, correction of any aortic valve insufficiency, and restoration of the dominant true lumen flow in the downstream aorta are crucial to successful treatment. Initial ascending aorta replacement with an open distal anastomosis under circulatory arrest with varying degrees of hypothermia and an adequate strategy of neuroprotection might be sufficient to save the patient's life. Nevertheless, an untreated false lumen in the distal aorta might jeopardize the benefit of this conservative strategy over the long term. ${ }^{1}$ Available data indicate that the size of the descending aorta at initial presentation, false lumen patency and diameter, along with primary and residual intimal tear location and size, are independent predictors of poor long-term outcome.

Recently, the progress in both operative and cerebral protection strategies along with evolving hybrid and stent graft technology paved the way for more aggressive approaches encompassing total arch replacement (TAR) combined with the frozen elephant trunk (FET) technique. Preexisting arch aneurysm, entry tear, or a large secondary tear occurring near the origin of supra-aortic branches or in the proximal descending aorta; circumferential dissection around the brachiocephalic vessels; and signs of distal malperfusion are commonly considered indications for extensive repair in selected patients. Of note, high-powered analyses of both the German Registry for Acute Aortic Dissection Type A and the International Registry of Acute Aortic Dissection support the idea that total arch repair is a significant predictor of increased operative mortality and morbidity. ${ }^{2}$ Nevertheless, such data are somehow biased by the unmeasurable individual patient frailty, the multifaced pattern of presentation of type A acute aortic dissection, and surgeon experience. Little is known about long-term benefits of this approach.

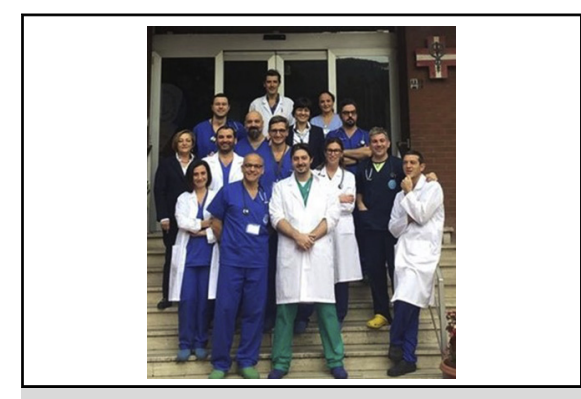

The cardiac surgery staff at Casa di Cura Montevergine, Mercogliano, Italy.

\section{Central Message}

Arch replacement with a stented elephant trunk has broadened the therapeutic armamentarium; nevertheless, technical refinements and better definitions of indications and long-term outcomes are needed.

See Article page 2267.
In this issue of the Journal, Shen and colleagues ${ }^{3}$ report outcomes of a remarkable series of 169 consecutive patients treated by TAR and FET. The study is representative of the emerging surgical standards in high-volume centers where an aggressive initial approach seems to be an institutional dogma. Between the lines, the search for the perfect surgical setup appears to be clearly underway. The authors aimed to disclose the predictors of postoperative oxygenation impairment, a frequent complication with profound prognostic implications. Intriguingly, the pathophysiology of postoperative oxygenation impairment is multifactorial and encompasses baseline respiratory function, direct effects of the dissection process on the inflammatory cascade, drawbacks of prolonged cardiopulmonary bypass and deep circulatory arrest, and factors related to blood product transfusion. Thus, hypoxemia is a surrogate marker of the full spectrum of patients' features, disease severity, complexity of surgery and the intensity of perioperative medical care.

The study results largely confirm previously reported evidence. $^{4}$ As expected, body mass index, preoperative homocysteine concentration, circulatory arrest time, and plasma transfusion were independent predictors of lung dysfunction, which was usually associated with generalized multiorgan dysfunction. Of note, a more complicated postoperative course was not associated with higher inhospital mortality rates. Another brick in the wall. Better definitions of indications, further technical refinements, 
and, above all, long-term outcomes are still needed to untangle this conundrum.

\section{References}

1. Smith HN, Boodhwani M, Ouzounian M, Saczkowski R, Gregory AJ, Herget EJ, et al. Classification and outcomes of extended arch repair for acute type A aortic dissection: a systematic review and meta-analysis. Interact Cardiovasc Thorac Surg. 2017;24:450-9.
2. Larsen M, Trimarchi S, Patel HJ, Di Eusanio M, Greason KL, Peterson MD, et al Extended versus limited arch replacement in type A aortic dissection. Eur J Cardiothorac Surg. 2017;52:1104-10.

3. Shen Y, Liu C, Fang C, Xi J, Wu S, Pang X, et al. Oxygenation impairment after total arch replacement with a stented elephant trunk for type-A dissection. $J$ Thorac Cardiovasc Surg. 2018;155:2267-74.

4. Girdauskas E, Kuntze T, Borger MA, Röhrich K, Schmitt D, Fassl J, et al. Acute respiratory dysfunction after surgery for acute type A aortic dissection. Eur J Cardiothorac Surg. 2010;37:691-6. 